\title{
Tris(2,4,6-trichloro-3,5-dinitrophenyl)methyl radical: a new stable coloured magnetic species as a chemosensor for natural polyphenols
}

\author{
Josep L. Torres, ${ }^{a}$ Begoña Varela, ${ }^{a}$ Enric Brillas ${ }^{b}$ and Luis Juliá ${ }^{* c}$ \\ a Departament de Química de Peptids i Proteïnes, Institut d'Investgacions Químiques i Ambientals (CSIC), \\ Jordi Girona 18-26, 08034, Barcelona, Spain \\ ${ }^{b}$ Departament de Química Física, Universitat de Barcelona, Martí i Franquès 1-11, 08028, Barcelona, \\ Spain \\ c Departament de Química Orgànica Biològica, Institut d'Investgacions Químiques i Ambientals (CSIC), \\ Jordi Girona 18-26, 08034, Barcelona, Spain. E-mail: ljbmoh@cid.csic.es; Fax: 349320459 04; Tel: 34 \\ 934006107
}

Received (in Cambridge, UK) 11th October 2002, Accepted 13th November 2002

First published as an Advance Article on the web 25th November 2002

We report the synthesis, electron paramagnetic resonance and electrochemical properties of a novel stable radical of the TTM series. Its strong electron acceptor ability has been tested with (-)-epicatechin, a natural polyphenolic antioxidant.

Organic radicals derived from the TTM series (tris $(2,4,6-$ trichlorophenyl)methyl radical (1)) are among the most stable carbon centered radicals due to steric hindrance of the substitutes around the trivalent $\mathrm{sp}^{2}$-carbon atom. ${ }^{1}$ However, they are very sensitive to redox processes and some of them are thus characterized as a new type of stable magnetic substrate exhibiting amphoteric redox behavior which make them good candidates to react by electron transfer reaction with either electron donating or accepting reactants to the corresponding carbanions or carbocations which have comparable stabilities. ${ }^{2}$ As these reactions can be monitored by electronic spectroscopy and electron paramagnetic resonance we are interested in exploring the potential of these radicals as chemosensors of natural and synthetic polyphenols. Redox properties of these radicals are chemically modulated with the introduction of different functional groups in the molecular structure either by replacement of the aromatic para-chlorines $1 b, c$ or by electrophilic substitution in the free aromatic meta-positions, ${ }^{3}$ in such a way that each radical species can show a different standard redox potential in cyclic voltammetry (CV). In this way, a list of redox chemosensors will be available to use, the suitable radical depending in each case on the electron donating properties of polyphenols.

The stable free radical DPPH has been widely used to conveniently test the free radical scavenging activity of the natural polyphenols by hydrogen abstraction reactions, generating relatively stable phenoxyl radicals which result in the formation of ortho-quinones. ${ }^{4}$ The method does not measure the capacity of natural polyphenols to participate in electron transfer reactions. However, carbon centered radicals derived from TTM do not directly abstract hydrogen due to steric factors and when the standard potentials of the radicals are appropriate, the reactivity will be based on the ability of the radical A to abstract an electron of the polyphenol $\mathrm{R}-\mathrm{OH}$ to generate transient radical cations which subsequently will evolve to stabilized phenoxyl radicals by removing a proton to the medium (Scheme 1). In this context, now we report a new free radical of the TTM series, the tris(2,4,6-trichloro-3,5-dinitrophenyl)methyl radical (2), its oxidant properties being analyzed in the presence of very diluted solutions of a natural polyphenolic antioxidant of the family of flavanols such as (-)-epicatechin.

For the preparation of 2, tris(2,4,6-trichlorophenyl)methane (3) 5 was converted by prolonged (6 days) and exhaustive nitration at $130{ }^{\circ} \mathrm{C}$ with fuming sulfuric acid and nitric acid (3:1) to tris(2,4,6-trichloro-3,5-dinitrophenyl)methane (4) $(32 \%)$. Treatment of the hydrocarbon precursor 4 with an

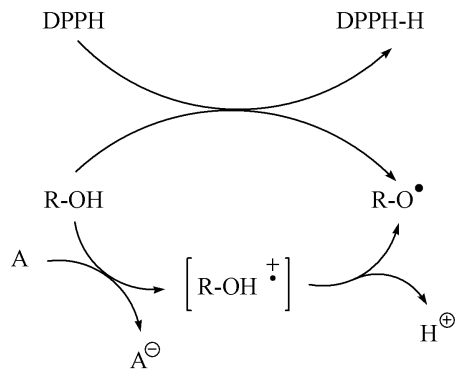

Scheme 1

aqueous solution of tetrabutylammonium hydroxide in THF in an equimolecular ratio and at low temperatures $(250 \mathrm{~K})(45$ $\mathrm{min})$ gave a red solution of the carbanion which is oxidized to radical 2 in the presence of an excess of 2,3-dichloro5,6-dicyanoquinone (Scheme 2). Highly diluted solutions of carbanion in THF are stable and the absorption spectrum consists of a broad band $\left(\lambda_{\max }=500 \mathrm{~nm} ; \varepsilon=23500 \mathrm{~L} \mathrm{~mol}^{-1}\right.$ $\left.\mathrm{cm}^{-1}\right)$.

Radical $\mathbf{2}$ is stable in solid form, and in chloroform solution in the dark its stability is $>99 \%$ after two days. The absorption spectrum of $\mathbf{2}$ is characterized by two typical radical bands (Fig. $1)$, with the second band showing hyperfine structure, at $\lambda(\varepsilon)$ in $\mathrm{nm}\left(\mathrm{dm}^{3} \mathrm{~mol}^{-1} \mathrm{~cm}^{-1}\right) 385(22600)$ and 513(740) and 560(800). These bands are bathochromically shifted relative to those of TTM radical (373, 478 and $542 \mathrm{~nm}$ ). As the stability of the radical series of TTM is mainly governed by the presence of the six ortho-chlorine atoms, all of which adopt a propeller-like conformation $^{6}$ with very similar torsion angles between the central carbon atom and the phenyl groups, the bathochromic shift is in correlation with the extended conjugation due to the nitro substitution in all meta-positions.

The cyclic voltammogram for the reduction of radical 2 in $\mathrm{CH}_{2} \mathrm{Cl}_{2}$ solution with tetrabutylammonium perchlorate $(0.1 \mathrm{M})$ as electrolyte on Pt displays a quasi-reversible $\left(E_{\mathrm{p}}{ }^{\mathrm{a}}-E_{\mathrm{p}}{ }^{\mathrm{c}}=\right.$

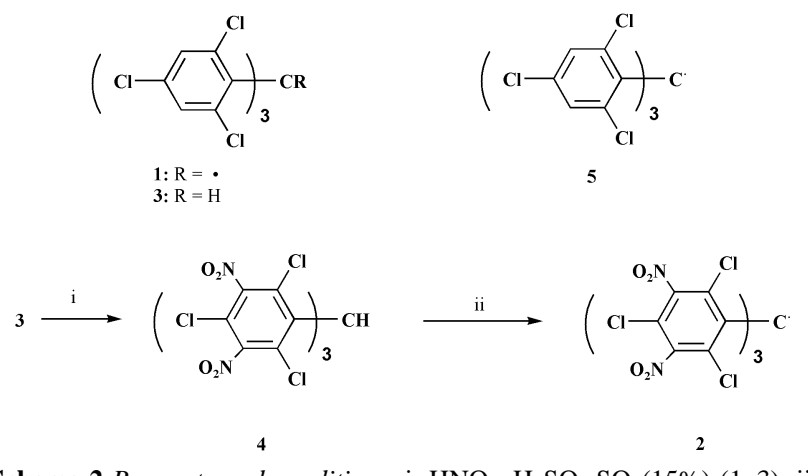

Scheme 2 Reagents and conditions: i, $\mathrm{HNO}_{3}: \mathrm{H}_{2} \mathrm{SO}_{4} \cdot \mathrm{SO}_{3}(15 \%)(1: 3)$; ii, (a) $\mathrm{Bu}_{4} \mathrm{NOH}-\mathrm{THF}$ (b) 2,3-dichloro-5,6-dicyanoquinone. 


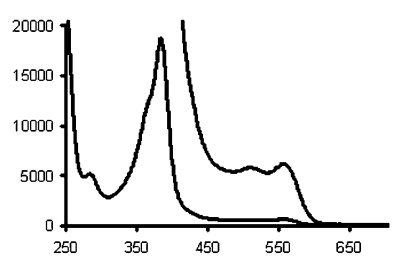

Fig. 1 Molar electronic absorption spectrum of radical 2 in $\mathrm{CHCl}_{3}$. The right-side of the graphic shows the molar absortivity enlarged by a factor of 10 .

$0.96 \mathrm{~V}$ at $v=200 \mathrm{mV} \mathrm{s}^{-1}$ and $0.78 \mathrm{~V}$ at $v=20 \mathrm{mV} \mathrm{s}^{-1}$ ) oneelectron redox pair corresponding to the process $\mathrm{A}^{\cdot}+1 \mathrm{e} \rightleftharpoons \mathrm{A}^{-}$ at a redox potential $\left(E^{0}\right)$ shown in Table 1 . The reversibility of this electrochemical process is indicative of the stability of both species. Redox values for the reduction processes of other stable radicals of the same triphenylmethyl series such as $\mathbf{1}$ and (4-amino-2,6-dichlorophenyl)bis(2,4,6-trichlorophenyl)methyl radical $(\mathbf{5})$ are also listed in Table 1.

Table 1 Redox potentials $\left(E^{0}\right)$ for the reduction of radicals $\mathbf{1}, \mathbf{2}$ and $\mathbf{5}$ $\left(\sim 10^{-3} \mathrm{M}\right)$ in $\mathrm{CH}_{2} \mathrm{Cl}_{2}$ solution with $0.1 \mathrm{M} \mathrm{Bu}_{4} \mathrm{NClO}_{4}$ on $\mathrm{Pt}$

\begin{tabular}{lr}
\hline Radical & \multicolumn{1}{c}{$E^{0 / \mathrm{V}}$} \\
\hline $\mathbf{1}$ & -0.66 \\
$\mathbf{2}$ & 0.58 \\
$\mathbf{5}$ & -0.83
\end{tabular}

a Potential vs. SSCE (NaCl-saturated calomel electrode).

It is worth emphasising the positive value of the peak potential for the reduction of $\mathbf{2}$ to its anion as a consequence of the strong electron acceptor properties of this radical. The remarkable shift in the redox potential of $\mathbf{2}$ relative to those of radicals $\mathbf{1}$ and $\mathbf{5}$ opens up the possibility of modulating the redox properties as a function of the different substituents in the aromatic structure of these open-shell species. Consequently, radical 2 unlike radical $\mathbf{1}^{7}$ is a good chemosensor of the presence of (-)-epicatechin. So, the oxidant ability of radical 2 as a good electron acceptor compound was evaluated by EPR, measuring the decrease of the intensity of the radical signal from a diluted solution $(100 \mu \mathrm{M})$ in choroform-methanol $(2: 1)$ in the presence of variable concentrations of (-)-epicatechin, and the results were plotted as the molar percentage of reduced radical against the concentration of (-)-epicatechin (Fig. 2). ${ }^{8}$

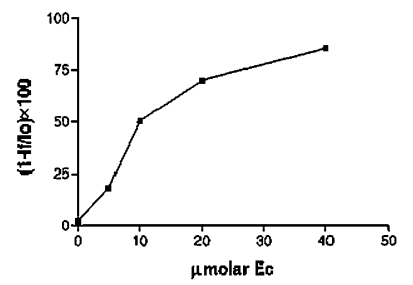

Fig. 2 Oxidant activity of radical 2 against (-)-epicatechin. The results are plotted as the molar percentage $\left(1-I_{\mathrm{f}} / I_{\mathrm{o}}\right) \times 100\left(I_{\mathrm{o}}\right.$ initial peak intensity of the radical signal in the EPR; $I_{\mathrm{f}}$ final peak intensity) of reduced radical from a $100 \mu \mathrm{M}$ solution in chloroform-methanol $(2: 1)$ in the presence of (-)-epicatechin at different concentrations.

$\mathrm{X}$-Band electron paramagnetic resonance (EPR) spectrum of radical 2 was recorded in degassed $\mathrm{CH}_{2} \mathrm{Cl}_{2}$ solution at room temperature and showed a narrow band $\left(\Delta H_{\mathrm{pp}}=0.4 \mathrm{G}\right)$ with non-resolved nitrogen splitting $\left(a_{6 \mathrm{~N}}<0.4 \mathrm{G}\right)$ (Fig. 3). At higher gain values, the isotropic coupling with the ${ }^{13} \mathrm{C}$ nuclear spins of the $\alpha$-carbon atom, the three bridgehead carbon atoms, and the six ortho-carbon atoms appeared in the spectrum on both sides of the main spectrum at approximately $30.0 \mathrm{G}, 13.0 \mathrm{G}$ and 11.4 G. ${ }^{9}$

We have reported the synthesis, and the electrochemical and magnetic properties of the stable triphenylmethyl radical 2.

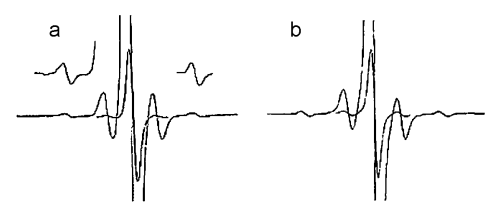

Fig. 3 (a) EPR spectrum of a solution of radical $2\left(\sim 6 \times 10^{-3} \mathrm{M}\right)$ in $\mathrm{CH}_{2} \mathrm{Cl}_{2}$ at room temperature (microwave power, $5 \mathrm{~mW}$; modulation frequency, 100 $\mathrm{kHz}$; modulation amplitude, 0.1 ) and amplification skowing ${ }^{13} \mathrm{C}$ couplings. (b) Computer simulation with the parameters given in the text.

Magnetic quenching of $\mathbf{2}$ by electron transfer reaction has been satisfactorily tested with (-)-epicatechin. As the antioxidant activity of natural polyphenols is closely related to their electron donating properties, ${ }^{10}$ this strategy opens up a new convenient way to evaluate natural polyphenols which are complements of the organism's antioxidant defence system. Work is now in progress to test the ability of this stable radical $\mathbf{2}$ and other related radical species with different plant polyphenolic antioxidants.

Financial support for this work from the MEC (Spain) through project BQU2000-0789-C02-02 and PPQ20000688-C05-03 is gratefully acknowledged. We also thank the EPR service of the Centre d'Investigació I Desenvolupament (CSIC) for recording the spectra.

\section{Notes and references}

$\dagger$ Selected data for 4 and 2. 4: IR (KBr) 3016(w), 1551(s), 1341(s), 1239(w), 1208(w), 1018(w), 949(m), 832(m), 783(m), 749(m), 631(m) $\mathrm{cm}^{-1} ;$ HRMS (EI): $m / z=823.675$, calc. for $\mathrm{C}_{10} \mathrm{HCl}_{10} \mathrm{~N}_{6} \mathrm{O}_{12}=823.679 .2: \mathrm{IR}(\mathrm{KBr})$ 1553(s), 1342(s), 1278(w), 1238(w), 1187(w), 946(w), 836(w), 787(w), 686(w) $\mathrm{cm}^{-1}$; HRMS (EI): $\mathrm{m} / \mathrm{z}=823.676(\mathrm{M}+\mathrm{H})^{+}$, calc. for $\mathrm{C}_{19} \mathrm{HCl}_{9} \mathrm{~N}_{6} \mathrm{O}_{12}=823.679$

1 (a) O. Armet, J. Veciana, C. Rovira, J. Riera, J. Castañer, E. Molins, J. Rius, C. Miravitlles, S. Olivella and J. Brichfeus, J. Phys. Chem., 1987, 91, 5608; (b) J. Carilla, Ll. Fajarí, L. Juliá, J. Riera and Ll. Viadel, Tetrahedron Lett., 1994, 35, 6529; (c) L. Teruel, Ll. Viadel, J. Carilla, Ll. Fajarí, E. Brillas, J. Sañé, J. Rius and L. Juliá, J. Org. Chem., 1996 61. 6063; (d) J. Carilla, Ll. Fajarí, L. Juliá, J. Sañé and J. Rius, Tetrahedron, 1996, 52, 7013

2 Preliminary results on cyclic voltammetry show the amphoteric character of TTM radical (1), (4-amino-2,6-dichlorophenyl)bis(2,4,6trichlorophenyl)methyl radical (5) and bis(2,6-dichlorophenyl)(2,4,6-trichlorophenyl)methyl radical exhibiting one reversible reduction and one reversible oxidation wave (E. Brillas and L. Juliá, unpublished results).

3 S. Salomé, J. Carilla, Ll. Fajarí, L. Juliá, E. Brillas and A. Labarta, Tetrahedron, 1995, 26, 7301

4 M. S. Blois, Nature, 1958, 181, 1199; W. Brand-Williams, M. E. Cuvelier and C. Berset, Lebensm.-Wiss. Technol., 1995, 28, 25.

5 M. Ballester, J. Riera, J. Castañer, C. Rovira and O. Armet, Synthesis, 1986, 64.

6 Attempts to get crystals for X ray analysis of radical 2 were unsuccessful. However, molecular structures of other radicals of the TTM series, such as radicals $\mathbf{1}^{1 a}$ and $\mathbf{5},{ }^{1 c}$ have confirmed that phenyl rings are twisted around their bonds to the trivalent carbon atom.

7 Radical 1 has shown to be stable in the presence of (-)-epicatechin in similar testing analysis.

8 During hydrogen transfer reactions from catechins and other flavonoids to radicals such as DPPH, two steps can be distinguished, a first fast step when the most labile $\mathrm{H}$ atoms are abstracted and a second slower step corresponding to the reaction of oxidation-degradation products. The byproducts may include partially inactivated polymeric species, particularly at high polyphenol concentration. This would result in doseresponse curves such as the one depicted in Fig. 2 for the electron transfer from (-)-epicatechin to radical 2. W. Brand-Williams, M. E. Cuvelier and C. Berset, Lebensm.-Wiss. Technol., 1995, 28, 25; O. Dangles, G. Fargeix and C. Dufour, J. Chem. Soc., Perkin Trans. 2, 1999, 1387; O. Dangles, G. Fargeix and C. Dufour, J. Chem. Soc., Perkin Trans. 2, 2000, 1653.

9 The spectrum was simulated following the WINSIM program provided by D. Dulog, Public EPR Software Tools, National Institute of Environmental Health Sciences, Bethesda, MD, 1996.

10 S. V. Jovanovic, S. Steenken, M. Tosic, B. Marjanovic and M. G. Simic J. Am. Chem. Soc., 1994, 116, 4846; S. V. Jovanovic, Y. Hara, S Steenken and M. G. Simic, J. Am. Chem. Soc., 1995, 117, 9881 